\title{
Debajo del radar. Los trabajos femeninos en los Altos de Jalisco
}

\author{
Patricia Arias* \\ Imelda Sánchez García** \\ Martha Muñoz Durán***
}

Recepción: 23 de marzo de 2018 / Aceptación: 25 de agosto de 2018

Resumen El objetivo de este artículo es describir y explicar los trabajos que han realizado y los que llevan a cabo las mujeres de los Altos de Jalisco en la actualidad, haciendo hincapié en las actividades femeninas que han existido, desaparecido o se han transformado y reaparecido en los espacios rurales y urbanos. El ejemplo de una de las regiones más tradicionales en términos socioculturales, pero también moderna e integrada en la economía globalizada, sirve para documentar las permanencias y persistencias, además de las transformaciones que han experimentado las sociedades rurales y los espacios urbanos. Se busca descubrir el papel de los salarios, y específicamente el de los ingresos femeninos que no se registran en la información censal ni en las estadísticas de empleos. Se trata de sacar a la luz los trabajos de las mujeres que operan al margen — debajo del radar — de la información que es detectada y cuantificada en los registros oficiales.

PALABRAS CLAVE: salarios, ingresos, trabajo femenino, trabajo masculino, grupos domésticos.

\footnotetext{
* Profesora investigadora en la Universidad de Guadalajara, Jalisco, México. mparias1983@gmail.com

** Estudiante de la Especialidad-Maestría en Producción Animal Sustentable en el Centro Universitario de los Altos de la Universidad de Guadalajara, Jalisco, México. isanchez@cualtos.udg.mx

*** Doctorante en Geografía y Ordenación Territorial en el Centro Universitario de Ciencias Sociales y Humanidades de la Universidad de Guadalajara, Jalisco, México.m_mduran@hotmail.com
} 


\section{Under the radar. Work by women in los Altos de Jalisco}

Abstract The aim of this article is to describe and explain the work that the women of los Altos de Jalisco have performed in the past, and what they are doing today. The article emphasizes female activities that have existed and sometimes disappeared over the years, or have been transformed and then re-appeared in rural and urban spaces. This example of female activities comes from one of the regions of the country that is among most traditional in socio-cultural terms, but is at the same time modern and integrated into the globalized economy. It serves to document the permanence and persistence experienced by rural societies and in urban spaces. There is a need to discover the role of salaries, and specifically that of female income, which are not recorded in the census or in statistics about employment. To put it another way, the paper is about bringing to light the work of women who operate on the sidelines, under the radar of the information detection and quantification recorded in official records. KEYWORDS: salaries, income, female labor, male labor, domestic groups.

\section{Introducción}

- lobjetivo de este artículo es describir y explicar los trabajos que han realizado, pero sobre todo que llevan a cabo las mujeres de los Altos de Jalisco en la actualidad. Se trata de descubrir el papel de los salarios, pero también de otros ingresos que no se registran en la información censal ni en las estadísticas de empleos. Y dicho de otro modo, se trata de sacar a la luz los trabajos femeninos que operan al margen — debajo del radarde la información que es detectada y cuantificada en los registros oficiales pero que tiene un enorme valor en términos de ingresos para los grupos domésticos, así como de las transformaciones suscitadas en la condición femenina.

Las investigaciones reconocen que la identificación y medición de las actividades donde la gente se emplea o autogestiona trabajos resulta complicada en términos cuantitativos (Gandini, Padrón y Navarrete, 2017). De hecho, se señala que a pesar de las modificaciones en las formas de medir que propuso la Conferencia Internacional de Estadísticas del Trabajo (С I E T) pueden quedar excluidos, entre otros, las mujeres, la población que vive en espacios rurales y quienes se dedican a la agricultura (Gandini et al., 2017).

A partir de esa constatación, uno de los intereses de este artículo es documentar, con materiales etnográficos recientes, la participación de las mujeres en empleos existentes, así como la búsqueda y creación incesante de trabajos que les permitan disponer de ingresos en efectivo de manera constante. 
Esto se debe a un gran cambio: el ingreso de las mujeres ya no es complementario al de los hombres, sino que se ha vuelto indispensable para ellas y para la integración de los presupuestos de los hogares de los que forman parte. En ese sentido, el trabajo femenino ha transitado del permiso a la obligación (Arias, 2016). Y ese cambio ha detonado transformaciones en la condición femenina, así como en las jerarquías tradicionales de los hogares.

Este artículo se basa en los materiales de investigación generados en los años 20142015, actualizados, en varios casos, a 2017 y 2018. Incluyó recorridos por las áreas rurales y urbanas de los municipios de las regiones Altos Sur y Altos Norte, que conforman la región Altos de Jalisco. Pero la mayor parte de la investigación se realizó en Altos Sur: trabajo de campo que supuso la aplicación de cuestionarios, la realización de entrevistas sucesivas y, sobre todo, la elaboración de 120 historias de vida de mujeres. La principal fuente de información han sido las propias mujeres; sus trayectorias, experiencias, relatos, reflexiones. En nuestro libro Quehaceres y obras. El trabajo femenino en los Altos de Jalisco (2015) se presentan, de manera sintética, 52 historias de vida de mujeres de la región.

Las entrevistas fueron realizadas a mujeres de todos los estados civiles — solteras, viudas, casadas, divorciadas - de diferentes edades, localidades y condiciones de vida. La información para una historia de vida requirió de al menos tres entrevistas. Pero también hay información de entrevistas que no se convirtieron en historias de vida. Las conversaciones y entrevistas fueron realizadas, en la mayor parte de los casos, en los hogares, que en muchas ocasiones coincidía con el lugar de trabajo. Cuidamos en todo momento que estuvieran solas para evitar interferencias e influencias de otras personas en sus relatos y reflexiones. Pero también buscamos y dejamos fluir diálogos entre mujeres de diferentes generaciones con el propósito de recuperar argumentos y explicaciones acerca de eventos y situaciones sucedidos en un mismo hogar.

En ocasiones, las tres pudimos realizar las entrevistas; en otras, fuimos dos, y también solo una de nosotras. De cualquier manera, los materiales de campo fueron compartidos y comentados entre las tres. La información fue recogida en diarios de campo y grabaciones que posteriormente fueron transcritas. La información acerca de salarios, condiciones de trabajo, opiniones y valoraciones sobre empleos, prestaciones y condiciones laborales provienen de la información generada en las mismas entrevistas y las historias de vida.

En 2016 realizamos otras dos investigaciones, que proporcionaron información novedosa sobre actividades económicas y empresariales, así como de cambios en la par- 
ticipación de las mujeres. Los resultados de esas investigaciones fueron «La evidencia del éxito. Residencias y mausoleos en Santiaguito, Arandas, Jalisco», de Martha Muñoz Durán e Imelda Sánchez García, y «Entre dos crisis. Los abarroteros de San Ignacio Cerro Gordo, Jalisco», de Patricia Arias, que se publicaron en 2017 en el libro colectivo Migrantes exitosos. La franquicia social como modelo de negocios.

\section{Sobre el trabajo}

La intensa migración campo-ciudad de la década de 1940 estuvo impulsada por el dinamismo del mercado de trabajo industrial, comercial y de servicios asociado al auge del modelo de sustitución de importaciones, que demandó miles de trabajadores en las ciudades (Bataillon y Rivière, 1973; Garza, 1980). Los trabajadores, muchos de ellos migrantes, se convirtieron en obreros en empresas que ofrecían salarios estables, prestaciones sociales y movilidad laboral que les permitieron insertarse, con más o menos suerte, como pobladores en la vida urbana.

Sin embargo, también se constató la emergencia de otra serie de empleos y actividades —informales se las llamó- donde imperaban otras condiciones: bajos ingresos, ausencia de prestaciones, inexistencia o incumplimiento de jornadas, falta de contratos, despidos injustificados; es decir, los trabajadores, a pesar de estar ocupados, recibían ingresos insuficientes y trabajaban de manera irregular y precaria (Jiménez, 2012; Roberts, 1978; Tokman, s.f.). Eso dio pie a la discusión en torno a las razones de la existencia y persistencia de mercados de trabajo duales: formal e informal. El debate, aunque intenso, no concluyó y ambos términos se usan todavía, aunque solo de manera descriptiva.

Porque la situación del trabajo ha cambiado de manera drástica. La dualidad de los mercados de trabajo, basada en las condiciones contrastantes entre ambos mercados, se ha desdibujado hasta casi desaparecer. Muchas actividades y empleos formales han adquirido rasgos tradicionalmente asociados a la informalidad: irregularidad, bajos salarios, contratos temporales, subcontratación (Jiménez, 2012).

En los establecimientos reconocidos — que serían «formales» en la anterior clasificación - predomina el empleo precario, de tiempo parcial, por hora, por cuenta propia, informal, inestable «como figuras que han desplazado al empleo asalariado remunerado (con contrato por tiempo indeterminado, estable, de jornada completa y con beneficios de seguridad social» (Gandini et al., 2017:18). Se ha observado además que las condiciones de trabajo de las mujeres son peores y las retribuciones que perciben son menores que las de los hombres (García y Oliveira, 2011). 
Se calcula que en la actualidad entre el $40 \%$ y $50 \%$ de las ocupaciones en América Latina se ubican en el antes llamado sector informal o en actividades de baja productividad, en las que los empleos son inestables y los salarios bajos (Jiménez, 2012). La mayor parte de los empleos que se han creado en América Latina han sido precisamente para trabajadores no calificados, con bajos salarios y sin acceso a prestaciones, seguro de salud o pensiones (García y Oliveira, 2011).

Frente a ese escenario, la Organización Internacional del Trabajo (о Iт) ha ampliado la noción de trabajo para incluir «todas las actividades realizadas por personas de cualquier sexo y edad con el fin de producir bienes o prestar servicios [...] independientemente de la legalidad de la actividad y de su carácter formal o informal $[\ldots] \gg y$ que «puede realizarse en cualquier tipo de unidad económica» (Navarrete y Padrón, 2017: 40-41).

Por lo anterior, en este artículo el trabajo es entendido como todas las actividades y quehaceres que llevan a cabo hombres y mujeres, dentro y fuera de los hogares, con el propósito de recibir salarios u obtener ingresos.

Entre 1995 y 2003 hubo un incremento de los trabajadores asalariados, pero en especial de las mujeres asalariadas (Pacheco, 2010). Para el siglo x x García (2010) documentó el mantenimiento, casi estancamiento, de las tasas de participación laboral masculina y el incremento sistemático de las tasas femeninas: de $71 \%$ en 1979 a $74 \%$ en 2006 en el caso de los hombres, en tanto que en el de las mujeres pasó de $21 \%$ a $39 \%$ en esos mismos años. Situación que se presentaba en todas las regiones, urbanas y rurales, del país. Desde la década de 1980 las investigaciones antropológicas y sociológicas dieron cuenta de la creciente incorporación de mujeres de los espacios rurales a los mercados de trabajo como resultado de la modernización, la apertura comercial, la globalización de las actividades agropecuarias y la introducción de nuevas actividades en el campo y las ciudades (Arias, 1988; García, 2010; González Montes y Salles, 1995).

El incremento de la participación laboral de las mujeres se ha dado sin modificar, de manera rotunda, una idea muy arraigada respecto al trabajo femenino: que el ingreso de la mujer solo es «complementario» para el presupuesto de los hogares de los que forman parte. Ese supuesto ha sido compartido por los miembros de los hogares, pero también por la academia y los empresarios.

La aceptación familiar, social y empresarial del carácter complementario de los ingresos femeninos se sustentaba en dos supuestos: en primer lugar, que los hogares podían sobrevivir con un solo ingreso, y en segundo, que el trabajo masculino era, a fin de 
cuentas, el que garantizaba la sobrevivencia económica de los grupos domésticos, lo cual les confería a los hombres el papel central e indiscutible de proveedores (Arias, 2016). Esto ya no es así. Se ha constatado que en la actualidad las mujeres «combinan en mayor medida la maternidad con el trabajo fuera de sus actividades domésticas» (García, 2010: 367), de manera que permanecen a largo plazo en los empleos asalariados o en los trabajos que autogeneran.

Los estudios documentan las razones por las que las mujeres han aceptado las condiciones laborales y salariales asociadas a los bajos ingresos. El argumento más repetido, en especial entre las que realizan trabajos en sus domicilios, es que son las actividades que les permiten combinar el cumplimiento de las obligaciones domésticas —irrenunciables e intransferibles - como madres, esposas e hijas con la presión económica para obtener mayores ingresos para sus hogares (Arias, 2009).

Sin embargo, tampoco es así. Nuestra hipótesis es que las actividades y características del empleo femenino deben ser vistas y entendidas como indicadores sensibles de al menos dos transformaciones importantes: en primer lugar, las características y tendencias actuales del empleo que han privilegiado la contratación de mujeres, y en segundo, transformaciones sociodemográficas que han alterado la conformación y dinámica de los hogares.

De esta manera, y solo de esta manera, se pueden entender y explicar las actividades bajo el radar que llevan a cabo las mujeres en los Altos de Jalisco. Nos detenemos, en especial, en la costura, que encubre muchos productos y quehaceres (bordado, deshilado, tejido, confección) que tiene una larga tradición en la región y que se ha mantenido, aunque transformada, y sigue siendo una opción de trabajo e ingresos para las mujeres.

\section{Los Altos de Jalisco}

En términos administrativos, los Altos de Jalisco se divide en dos regiones: Altos Norte, integrada por ocho municipios, y Altos Sur, por doce (véase mapa 1). La región Altos Norte está conformada por Encarnación de Díaz, Lagos de Moreno, Ojuelos, San Diego de Alejandría, San Juan de los Lagos, Teocaltiche, Unión de San Antonio y Villa Hidalgo. La región Altos Sur por Acatic, Arandas, Cañadas de Obregón, Jalostotitlán, Jesús María, Mexticacán, San Ignacio Cerro Gordo, San Julián, San Miguel el Alto, Tepatitlán de Morelos, Valle de Guadalupe y Yahualica de González Gallo. La región colinda con la zona metropolitana de Guadalajara (zMG) y con los estados de Aguascalientes, Guanajuato y 


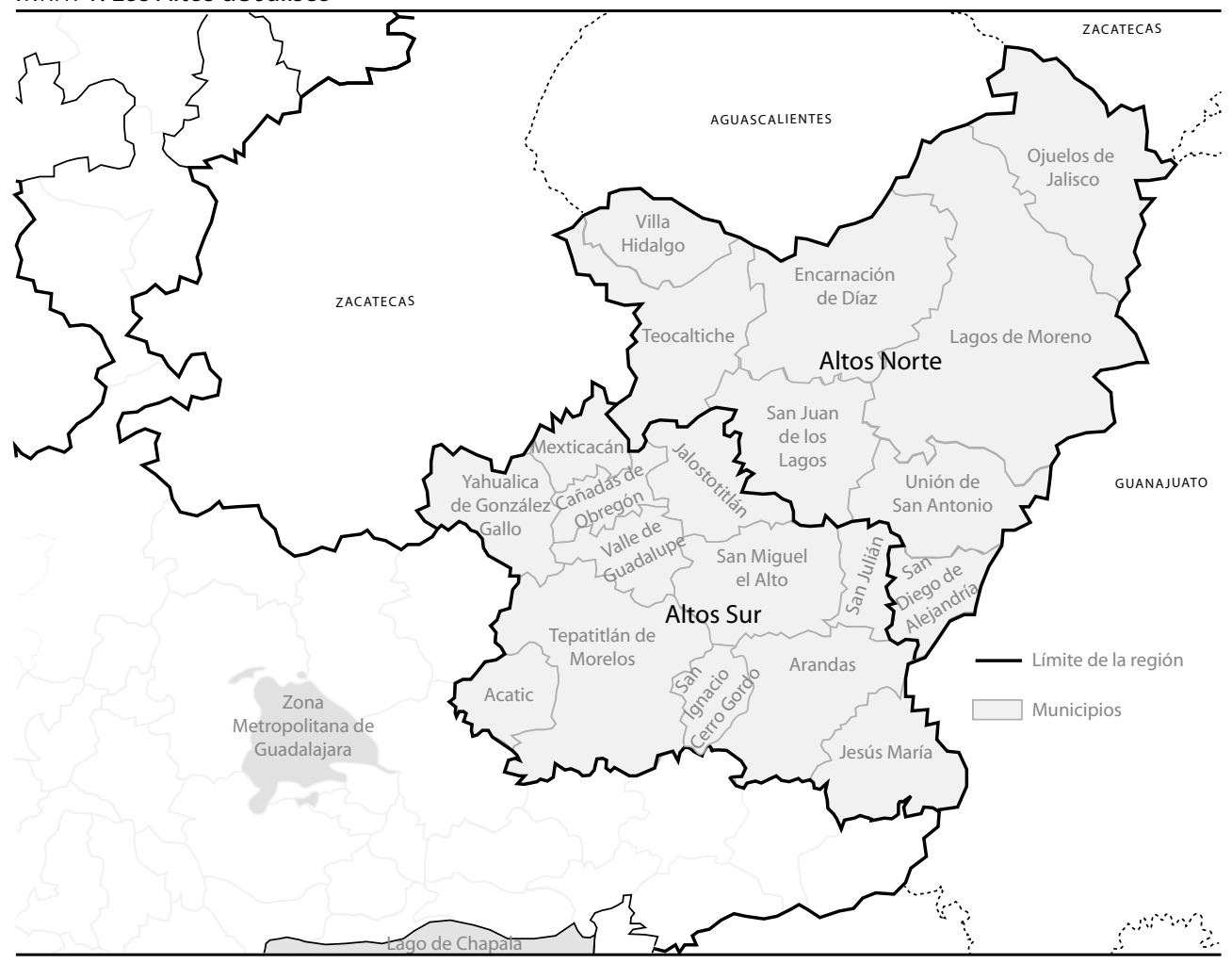

Zacatecas, con los cuales los municipios fronterizos suelen compartir actividades y mantener relaciones significativas.

De acuerdo con información de la Encuesta Intercensal 2015, las regiones Altos Sur y Altos Norte sumaban 807141 habitantes, lo que representaba una décima parte de la población del estado de Jalisco (10.2\%). A los Altos los han definido tradicionalmente cinco características: una sociedad de economía ganadera y lechera basada en explotaciones de pequeña y mediana escala; asentamientos rurales en forma de ranchos dispersos donde la gente vivía y trabajaba; predominio de la propiedad privada; migración laboral masculina centenaria a Estados Unidos; una tradición, también centenaria, de trabajo femenino en los hogares, en especial la cría de pequeñas especies (pollos, puercos), la elaboración de productos lácteos y, sobre todo, la costura (Arias, 2016).

Actualmente, se la reconoce como una región agropecuaria que ocupa los primeros lugares a nivel nacional en la producción de leche, huevo, carne de cerdo y pollo; por la 
existencia de actividades manufactureras en casi todos los municipios; por una intensa fragmentación de la propiedad; por una notable urbanización y conectividad entre las localidades y municipios; por la cancelación de la migración a Estados Unidos como opción laboral generalizada, y por la intensificación y generalización del trabajo femenino asalariado fuera del hogar. En lo que se refiere a empleo, los Altos constituye una excepción respecto a otras regiones de Jalisco porque allí existe un amplio abanico de fuentes de trabajo para la población, en especial para la femenina.

En 2014 Jalisco ocupó el primer lugar en la producción de carne de cerdo y pollo. La mayor producción de ambas se concentra en los Altos. En 2015 la producción jalisciense de bovinos representó casi una quinta parte (19.64\%) de la producción nacional, una proporción similar a la de porcinos (19.27 \%) y las de huevo para plato y carne de pollo representan más de una cuarta parte (25.93\%). En la región, en 2015, había 18732 unidades de producción, es decir, granjas de producción de huevo para plato y carne de pollo, y 9388 de porcinos. La mayoría de las empresas tienen establecimientos en diferentes lugares de la región y ofrecen servicio de transporte, de manera que en una misma granja trabajan mujeres de diferentes ranchos, pueblos y ciudades.

El crecimiento y la modernización de la porcicultura y la avicultura, asociadas a la segmentación de los procesos productivos y la dispersión de los establecimientos por la geografía regional, requirieron de muchos trabajadores, y las mujeres se incorporaron a la oferta laboral en las granjas de cerdos, aves de postura y pollos de engorda. Más de la mitad (entre $60 \%$ y $80 \%$ ) de los trabajadores de las granjas avícolas son mujeres, y en la porcicultura puede llegar hasta a la mitad (40-50\%).

Además, en cada municipio existen fábricas y talleres que emplean mujeres. Las empresas, diseminadas por el espacio regional, tienen que ver con la actividad agropecuaria y agroindustrial: productos lácteos, champiñonera, deshidratadoras de huevo y una gran cantidad de fábricas de tequila. Pero también existen establecimientos donde se elaboran prendas de vestir, blancos, calzado, botanas, cajetas, conos de helado, cucharas, dulces, globos, embotelladoras, huaraches, mochilas. Desde 2010 resurgió la actividad maquiladora, la cual tiene que ver con el bajo costo de la mano de obra en México, que ha vuelto a hacer rentable la producción nacional de calzado, prendas de vestir y una gran variedad de artículos textiles y de piel.

Un poco de historia

Diversificación y género. Desde fines del siglo XIX se dejó sentir en la región la necesi- 
dad de ingresos económicos distintos y complementarios a las actividades agropecuarias tradicionales: agricultura y ganadería de bovinos (Rollwagen, 1968; Taylor, 2013). La necesidad de ingresos llevó a la diversificación de actividades y la migración. Desde la década de 1920, han señalado Díaz y Estrella (1979), se advertía lo que ellos definieron como una crisis ecológica que afectaba la viabilidad de las pequeñas explotaciones agroganaderas y obligaba a los vecinos a salir en busca de trabajo e ingresos: los hogares eran numerosos; la tierra, el principal recurso, era de mala calidad y había sido intensivamente trabajada; la propiedad había estado sometida, generación tras generación, a procesos de subdivisión. Lo anterior había dado lugar al predominio de la renta de tierras y la mediería como sistema de organización de la producción ganadera; procesos, ambos, que impulsaban la migración (Rollwagen, 1968; Taylor, 2013).

En los Altos la diversificación de ingresos dio lugar a fenómenos espacializados, jerarquizados y segmentados por sexo. A fines del siglo x Ix la construcción de las vías de ferrocarril hacia la frontera norte y la demanda de trabajadores para las labores agrícolas en el sur de Estados Unidos detonaron un fenómeno inédito pero que cobró enorme fuerza en la región: la migración masculina de larga distancia (Durand y Arias, 2004). La migración laboral a ese país se articuló con la necesidad de ingresos que las actividades agroganaderas de la región no garantizaban. La migración se convirtió en una exigencia para que los hombres pudieran cumplir con la obligación de ser proveedores de sus hogares (Arias, 2016).

La movilidad no era para las mujeres, aunque también tuvieran necesidad de ingresos. Para ellas era muy difícil salir de sus hogares. El poblamiento en ranchos dispersos, la inexistencia de actividades que demandaran sus servicios y el escaso apoyo que requerían las actividades agroganaderas orientaron las habilidades y los afanes femeninos hacia otros derroteros; es decir, hacia actividades que podían desempeñar en sus casas y solares. Desde la época del Porfiriato, gracias al paso del ferrocarril, se incrementó la demanda de huevos y las mujeres se convirtieron en expertas criadoras de gallinas; también elevaron su participación en la elaboración de quesos, otro producto que, gracias al ferrocarril, empezó a llevarse en grandes cantidades a la Ciudad de México. Las mujeres eran retribuidas en efectivo, pero también aceptaban artículos y productos «industriales» que se necesitaban en los hogares, que no eran muchos: telas e hilos, velas y cerillos, jabón.

La especialización porcícola de La Piedad, Michoacán, microrregión fronteriza con los Altos de Jalisco, hizo posible que las mujeres de ranchos y pueblos alteños se hicieran 
cargo de la cría de lechones desde que nacían hasta que alcanzaban el peso de diez kilogramos, momento en que eran trasladados a las granjas de engorda.

La otra gran tradición de trabajo femenino en los hogares, rurales y urbanos, ha sido la costura (De Anda, 1989; Yáñez, 1947, 1962). Llamamos costura a todas las técnicas y productos que contengan elementos de bordado, confección, deshilado y tejido.

La producción hogareña de artículos y prendas a cargo de las mujeres tenía una importancia crucial para el abasto familiar y los intercambios sociales. Los productos servían de regalo para los matrimonios, cuando nacían bebés, para las fiestas de la iglesia, para agasajar al sacerdote recién llegado; para acompañar otros regalos. Entre las casas y los ranchos había un tráfico incesante de servilletas que resguardaban otros intercambios.

La confección de la ropa y todos los enseres de los hogares corrieron, hasta principios del siglo xx, por cuenta de las mujeres de una casa. Ellas compraban las telas, en especial la manta, y los hilos, a partir de los cuales confeccionaban la ropa cotidiana de los hombres, de ellas mismas, de los hijos e hijas, de los bebés, y todos los artículos que se requerían en los hogares. La fiesta patronal era la única ocasión en que se recurría a las modistas y costureras para «estrenar».

Cada mujer aportaba al matrimonio un ajuar hecho por ella misma que comprendía: juegos de sábanas y almohadones bordados; manteles, servilletas para las tortillas, llamados semanarios, carpetas para adornar muebles y cubrir recipientes y productos. Poco después del matrimonio tendría que demostrar sus habilidades en la hechura de todo lo necesario para los bebés que, muy pronto, comenzarían a nacer.

Esa habilidad se convirtió en la principal manera de obtener ingresos en efectivo. En la década de 1960 se dejaron sentir cambios económicos, demográficos y socioculturales: intensificación de la crisis de las actividades agropecuarias tradicionales, incorporación de productos industriales al consumo de los hogares, gastos asociados a la educación de los hijos (transporte, útiles escolares, alimentos), migración de las familias de los ranchos a las cabeceras municipales.

Frente a esos cambios, las mujeres incrementaron el tiempo que dedicaban a la elaboración de artículos de costura, que les permitían acceder con rapidez a ingresos en efectivo. En las cabeceras municipales había tiendas y señoras que recibían la producción de costura doméstica y la llevaban o enviaban a mercados donde era muy bien cotizada por la calidad de los bordados y acabados. Los establecimientos comerciales de las ciudades de México, Guadalajara, Guanajuato y Zacatecas recibían con agrado y sin límites los juegos de sábanas, ajuares de bebé, servilletas, carpetas y manteles que se producían en 
grandes cantidades. Además estaba el enorme mercado de San Juan de los Lagos, visita obligada de la grey católica al santuario de la Virgen de esa ciudad, donde la costumbre obliga a los peregrinos a comprar todo tipo de objetos y prendas para regalar o vender en sus comunidades o en Estados Unidos.

La modalidad predominante era que las mujeres - madre e hijas, hermanas y cuñadas - cosieran, bordaran y tejieran las diferentes partes de un artículo y lo entregaran lavado, almidonado y planchado, es decir, listo para su venta, a las comerciantes. Las productoras compraban la tela, los hilos y decidían las técnicas, diseños y colores de los bordados. Se trataba de un amplio sistema de trabajo familiar a domicilio emanado de las propias trabajadoras en busca de ingresos. También había comerciantes que entregaban los materiales a mujeres de los ranchos para que les confeccionaran productos. Las comerciantes, con contactos en diferentes mercados, recuperaban y potenciaban ese trabajo a domicilio, disperso, pero independiente.

La migración masculina a Estados Unidos, predominante hasta fines del siglo $\mathrm{xx}$, contribuyó, si no a la emergencia, sí a la persistencia y renovación del trabajo femenino en los hogares. Ante la ausencia temporal de padres y esposos, la disponibilidad de tiempo y la necesidad creciente de dinero en efectivo, las mujeres aceptaban encargos en sus hogares, en ocasiones sin que los esposos lo supieran. Ellas solían dejar de trabajar en las temporadas anuales, en los dos o tres meses que los maridos regresaban a casa. Y luego reiniciaban los trabajos de la costura.

De esa manera, el trabajo femenino en los domicilios pudo permanecer tan socialmente invisibilizado que no afectaba el principio patriarcal de que los hombres eran los proveedores de los hogares. En muchos casos, los ingresos femeninos de la costura aceleraron incluso el cumplimiento de las metas migratorias (construcción de la casa, compra de tierra, animales, vehículos), lo que permitía reducir el tiempo que los migrantes permanecían en Estados Unidos.

Se puede decir, entonces, que en casi todos los municipios de los Altos de Jalisco ha existido una larga y vigorosa tradición de diversificación de actividades e ingresos económicos, que ha estado pautada por una clara y consistente división sexual y espacial del trabajo basada en la movilidad de los hombres y el trabajo a domicilio para las mujeres, en la cría de animales domésticos y la manufactura relacionada con la costura.

La industrialización que no prosperó. La tradición de la costura en los Altos, ampliamente conocida en los mercados urbanos, dio lugar a la llegada de un tipo particular de em- 
presas a la región: las maquiladoras. En las décadas de 1970 y 1980 hubo una oleada de deslocalización de industrias urbanas dedicadas a la producción de bienes de consumo. Frente a la inminente competencia que se anunciaba con la firma del Tratado de Libre Comercio ( $\mathrm{T}$ LC), la necesidad de abaratar costos y eludir controles (impuestos, sindicatos, salubridad), hubo empresas urbanas que decidieron desconcentrar y fragmentar la producción en el espacio rural. Así, se instalaron talleres en los Altos, en ocasiones grandes, de maquila de prendas de vestir, calzado, esferas de Navidad, guantes, globos (Arias, 1988; Arias y Wilson, 1997).

Eso animó a gente de las localidades, en especial a las mujeres, a dedicarse también a la fabricación; en ocasiones como maquiladoras de empresas, en otras de manera independiente, es decir, como propietarias de talleres. El crecimiento urbano y la importancia de la moda como principal tendencia del consumo de prendas y artículos de casa había abierto una ventana de oportunidad para la confección industrial de sábanas, colchas, almohadones, prendas de vestir femeninas en establecimientos que reunían trabajadoras y que, de manera eventual o permanente, enviaban parte de la producción a maquilar a otros talleres y al trabajo a domicilio. Entre 1960 y 1980 proliferaron fábricas de diferente tamaño, talleres y hubo abundante trabajo a domicilio en pueblos y ranchos (Arias y Wilson, 1997). Las dueñas de talleres les prestaban las máquinas a las trabajadoras a domicilio y las animaban a que las compraran con la seguridad de que tendrían trabajo. Así, llegaron a los hogares rurales y a las incipientes colonias populares de las ciudades de la región máquinas industriales de coser y de bordar (Arias y Wilson, 1997; Wilson, 1990).

Así se difundió también, en cabeceras municipales y ranchos, el trabajo a domicilio para la costura y el acabado de calzado, cinturones, adorno de sombreros de charro, fabricación de esferas navideñas de vidrio soplado, confección de guantes industriales, dulces, muebles de jardín. Eran talleres maquiladores de empresas mayores o de empresarios y empresarias de la región que desarrollaron sistemas independientes de comercialización regional y nacional.

Sin embargo, la industrialización rural a cargo de empresarios locales no prosperó. La llegada de productos extranjeros, en especial chinos y coreanos, que arribaron arropados por la apertura comercial, pero también por el contrabando, llevaron a la desaparición de las empresas de la costura, así como de otros giros, y de la red de trabajo que habían tejido en la región. Algunas empresarias vieron venir la catástrofe y se retiraron a tiempo, otras muchas tuvieron que cerrar cuando les fue imposible continuar. 
Como resultado de ese proceso hubo un decremento del empleo manufacturero femenino de bienes de consumo hasta la década de 2010 .

\section{Los trabajos de las mujeres hoy}

En la actualidad, las producciones domésticas de pollo, huevo y la cría de lechones han dejado de ser generadoras de ingresos para las mujeres en sus hogares. Ya no es una práctica que se realice a nivel doméstico.

La exitosa especialización pecuaria de los Altos incrementó la demanda de trabajadoras y el empleo femenino en la avicultura y la porcicultura. Ambos sistemas de producción operan en escalas industriales especializadas y segmentadas, en granjas donde se contrata a mujeres como trabajadoras asalariadas.

Una actividad tradicional de las mujeres que sí se ha revitalizado es la fabricación de quesos y productos lácteos artesanales, es decir, a escala doméstica, fabricados con leche sin pasteurizar. La demanda de quesos de «rancho» de buena calidad, no industriales, en las ciudades y espacios como la extensa zona metropolitana de Guadalajara ha permitido a las mujeres retomar, con gran éxito, la fabricación de diversos quesos, a lo que se ha añadido la elaboración de crema, mantequilla, requesón y yogurt. No es que antes no se hicieran, lo que ha cambiado es la escala de la demanda. La fabricación de quesos y productos lácteos está asociada a pequeños ranchos de ordeña donde el excedente de litros de leche es utilizado por las mujeres, por lo regular la esposa o alguna hija del dueño del rancho, y es una buena manera de rentabilizar mejor la leche.

La buena calidad del queso de alguna productora suele detonar la ampliación de su mercado, lo que puede llevarla a comprar leche de otros establos para una mayor producción. Los quesos artesanales se mueven en circuitos microrregionales: clientes individuales, clientes fijos de tiendas de abarrotes, fondas y mercados o bien comerciantes que los llevan a vender a tianguis, mercados, tiendas de abarrotes de otros lugares. Se trata de una actividad que se mantiene estable en términos de la envergadura de los negocios y el número de productoras y comerciantes. Las fabricantes suelen ser de dos tipos: señoras que siempre lo han hecho o mujeres de ranchos que así obtienen ingresos en efectivo de manera regular. En cualquier caso, no se observa mayor interés de las mujeres jóvenes por dedicarse a esta actividad, menos aún en los espacios urbanos.

Una actividad reciente, que se realiza más en la calle que en los domicilios, es la limpieza de bolsas y envolturas de plástico. Aunque la realizan algunos hombres, en especial ancianos, es una actividad que también llevan a cabo mujeres jóvenes. En la ciudad 
de Tepatitlán y en Santiaguito, municipio de Arandas, existen espacios donde se apilan bolsas sucias a las que las mujeres les quitan los objetos que puedan tener —etiquetas, cintas, restos de comida-, les sacuden la mugre y las secan al sol, para luego colocarlas en contenedores de plástico a los que llaman jumbo. Esa limpieza es imprescindible para que el material plástico pueda ser procesado en las fábricas de reciclado, que lo convierten en mangueras y bolsas negras para basura. El kilo de plástico «acomodado», es decir, limpio, se paga a 1.20 pesos y cada trabajadora puede limpiar, máximo, entre 100 y 200 kilos al día. Eso le permite un ingreso de 120 a 240 pesos diarios. Las trabajadoras no utilizan guantes ni ningún tipo de protección. Pero dicen que nunca les ha pasado nada.

\section{Comidas, antojos y golosinas}

Como se ha mencionado, en la región existe una amplia oferta de trabajo para las mujeres en granjas de pollo y cerdo, fábricas y talleres. La expansión del empleo fuera de los hogares ha impulsado el crecimiento del trabajo femenino en el comercio y los servicios, en especial, aunque no únicamente, en las cabeceras municipales. La salida cotidiana de hombres y mujeres de sus casas, entre otras cosas, ha modificado los hábitos de consumo de la población. La proliferación de establecimientos de comida está asociada a cambios sociales y laborales que han alterado los ritmos y las rutinas tradicionales de la alimentación en los hogares: los horarios laborales corridos, las mujeres que salen a estudiar y trabajar todos los días, han reducido el personal y el tiempo destinado a la preparación doméstica de alimentos, así como la posibilidad de regresar a comer a las casas. La alimentación de trabajadores y estudiantes depende cada vez más de la oferta que se despliega en las calles. Sobre todo, en la noche nadie puede, nadie quiere llegar a la casa, después de una jornada laboral o estudiantil, a preparar platillos complicados. Es mucho más sencillo -y menos conflictivo - apelar a la variada y barata oferta callejera.

En general, el ancho mundo de la oferta de comida pertenece a las mujeres. Ellas, en sus hogares preparan, durante todo el año, diferentes tipos de dulces, pasteles, tamales, dulce de calabaza, cuecen elotes; hacen ensaladas, gorditas de maíz, duritos, tacos dorados. En temporada de calor elaboran bolis, hielitos, nieves raspadas, mangonadas (paletas de hielo con mango molido, pero también de otras frutas) que se conservan en refrigeradores domésticos. Los sistemas de venta pueden ser diversos y, muchas veces, complementarios. Hay señoras que salen a vender elotes y tamales por las calles, otras los ofrecen en su casa. La fabricación de pasteles, asociada a temas de caricaturas infantiles y 
otros personajes, se ha convertido en un nicho importante de trabajo femenino a domicilio. Las que los hacen tienen catálogos, que se promueven en Facebook y WhatsApp, donde es posible escoger diseños originales para pasteles de bautizo, boda, cumpleaños, primeras comuniones, $\mathrm{XV}$ años, posadas.

En las calles, sobre todo en las proximidades de centros de trabajo, salud y educación, hay preparación y venta de alimentos desde las mañanas: café, jugos, quesadillas, gorditas, tacos de olla. A mediodía se ofrecen comidas caseras y lonches. En la tarde se ofertan dulces, duros, salchichas, bolis. La oferta gastronómica se amplía aún más en la noche: señoras que en las cocheras y puertas de sus casas venden tacos, pozole, sopes, tamales, enchiladas, tamales en caldito, tacos dorados, hot-dogs.

También los domingos prospera la venta de alimentos y golosinas. Abundan las señoras que habilitan cocheras y cuartos para vender menudo y, todo el día, en el centro de cualquier población, se ofrecen infinidad de golosinas elaboradas para la ocasión en los hogares. La localización es crucial. Vivir o poder colocarse cerca de lugares transitados como plazas, mercados, escuelas, hospitales y templos garantizan la presencia de clientes en diferentes días y horarios.

Aunque en los ranchos hay menos posibilidades para los negocios de comida, las mujeres no dejan pasar la oportunidad de preparar alimentos o golosinas para los trabajadores en los lugares donde se han instalado industrias, granjas de pollos o cerdos o donde se realiza alguna obra pública.

\section{Las ventas}

La otra actividad que ha experimentado un notable incremento son las ventas a domicilio de todo tipo de productos artesanales e industriales, muchos de los cuales se ofrecen por catálogo. Existen dos modalidades. Por una parte, el trabajo dependiente que aparece encubierto en la figura de comisionistas, es decir, personas que reciben un porcentaje por las ventas que realizan. Empresas internacionales y nacionales han desarrollado sistemas de venta a domicilio a través de redes de mujeres que venden casa por casa. Es el caso de Betterware, Omnilife, Tupperware, Yakult. Pero también hay fábricas y establecimientos comerciales de ropa, calzado, lencería, cosméticos, perfumes, colchas, sábanas, edredones, accesorios, bisutería, joyería que basan su comercialización, o parte de ella, en las ventas a domicilio a cargo de comisionistas.

La otra modalidad es la venta a domicilio independiente, es decir, de señoras que por su propia cuenta, gracias a algún contacto o familiar, incursionan en la venta de los 
artículos ya mencionados. En los últimos años, la inseguridad ha hecho decaer el negocio de joyería, en especial, el de joyería fina. Pero ha hecho prosperar otros.

Hay señoras que compran prendas de vestir, en especial para mujeres y niños, en centros especializados de producción de ropa de moda, como las ciudades de Villa Hidalgo y Zapotlanejo, en Jalisco, o la calle Medrano, en Guadalajara; de zapatos y tenis de León, Guanajuato, que luego ofrecen al menudeo en sus casas y en camionetas especialmente habilitadas que recorren barrios y colonias populares.

Al mismo tiempo, han proliferado los bazares de ropa y enseres en cocheras y camionetas. Allí se ofrece ropa de segunda mano de Estados Unidos, que se compra por pacas en la frontera y se vende por pieza en México. Hay mujeres que viven en Estados Unidos que traen ropa nueva y de segunda mano para que las vendan sus familiares en la región y se reparten las ganancias. Hay señoras que distribuyen a otras mujeres ropa y calzado usado de Estados Unidos para su venta en ranchos. Esta actividad ha aumentado tanto en los últimos años que las ventas de cada establecimiento se han reducido.

Las ventas a domicilio tienen dos ventajas para las compradoras: los productos son pagados en abonos, es decir, con crédito, y es una manera accesible de estar a la moda, algo cada vez más necesario en los mercados de trabajo femeninos. Para las familias pobres, es una manera de acceder a prendas de vestir de segunda mano de bajo precio.

Y, por supuesto, existen señoras que organizan tandas, esa forma de ahorro tan difundida para generar montos de dinero que les permiten planear gastos y hacer inversiones a mediano plazo. En la región existe la modalidad de tandas de productos — colchas y edredones-, pero también de artículos de valor equivalente que se entrega a las diferentes participantes de la tanda.

\section{Los servicios}

A los empleos tan conocidos como el servicio doméstico, la limpieza de casas por horas, lavado y planchado de ropa, se han agregado actividades como corte, peinado y teñido de pelo, arreglo de uñas, masajes, limpieza facial. Son quehaceres que se ejercen en los domicilios y se adaptan a los horarios de las clientas, muchas de ellas trabajadoras que no pueden acudir a los establecimientos en los horarios comerciales convencionales. Es decir, pueden ser atendidas en la tarde-noche, cuando salen de trabajar o durante los fines de semana.

Pero lo más novedoso y generalizado de los últimos tiempos es el cuidado de niños, en especial bebés, y de ancianos. Esas tareas del cuidado recaían tradicionalmente en las 
mujeres que, como madres, hijas, hermanas, eran la que se encargaban de atender a los bebés en los hogares. Era parte de las obligaciones de las mujeres por la cual no existía una retribución monetaria. Eso ha cambiado. Con la disminución del número de hijos en los hogares, la incorporación de las mujeres al trabajo y la prolongación de los años de estudio, los hogares ya no disponen de tantas mujeres para esa tarea ni es posible que la ejerzan de manera gratuita.

Porque ellas ahora pueden trabajar fuera del hogar en establecimientos formales con horarios establecidos. Así las cosas, las trabajadoras deben contar con alguien que se encargue de sus hijos, sobre todo cuando son bebés, es decir, hasta que puedan enviarlos a la guardería o al kínder. Esa situación ha detonado un nuevo mercado de trabajo a domicilio para mujeres casadas, mayores o con hijos pequeños que se dedican de tiempo completo a dar ese servicio. En sus casas reúnen entre dos y seis bebés. Los horarios y precios son muy variables, pero por una jornada de seis horas de lunes a viernes se pagan entre $500 \mathrm{y}$ 1200 pesos semanales por niño. Pero también se ofrecen servicio por horas. En ese caso, se cobra 10 y hasta 20 pesos por hora. También se cobra por el servicio de recoger a los niños en las escuelas y esperar a que lleguen los padres.

La cuidadora puede ser madre, hermana, cuñada, sobrina, suegra de la trabajadora. Pero tiene que pagarle. En los Altos, a diferencia de otras regiones, el dinero para el pago de la cuidadora sale de los ingresos de ambos cónyuges. O quizá eso depende del estrato socioeconómico de la pareja. De cualquier manera, es ella la que tiene que negociar y renegociar los arreglos para el cuidado de los hijos, que suelen ser muy cambiantes.

Lo más novedoso y complejo es el cuidado de los ancianos, que recaía tradicionalmente en los hijos, en especial, las hijas. Pero ha habido muchos cambios. En general, se ha reducido el número de hijos e hijas en los hogares, pero además se ha modificado el escenario del cuidado: se han prolongado los años de vida de los padres, pero en calidad de enfermos, muchos con padecimientos crónicos a largo plazo que resultan costosos; se ha generalizado la necesidad de las mujeres de trabajar y conseguir ingresos y se ha intensificado la migración femenina por tiempo indefinido a Estados Unidos.

Las mujeres que trabajan ya no están, como antes, disponibles de tiempo completo para hacerse cargo de la atención de padres ancianos y enfermos. Ellas deben combinar las exigencias del trabajo con las obligaciones domésticas y el cuidado de los ancianos. Pero los ingresos locales son insuficientes para sufragar los gastos del cuidado y los cuidadores. A partir de la amnistía otorgada por la Ley de Reforma y Control de Inmigración (I R C A, por sus siglas en inglés) en 1986, se desencadenó el proceso de reunificación familiar que 
hizo que las mujeres comenzaran a emigrar, como nunca antes, para reunirse con los maridos y padres que habían conseguido regularizar su estancia en Estados Unidos (Durand y Massey, 2003). Se incrementó también la migración femenina indocumentada. Desde 2005 ha resultado cada vez más difícil que las mujeres regresen a sus lugares de origen, incluso en los casos de enfermedad de los padres; menos aún las indocumentadas.

Las que tienen documentos por lo regular trabajan y no pueden dejar sus empleos o pedir permisos prolongados, y las indocumentadas, que también trabajan, no pueden arriesgarse a regresar a cuidar a sus padres ante el riesgo de no poder retornar a Estados Unidos, donde están sus esposos e hijos. Los casos de hijas que lo hicieron y no pudieron ingresar de nueva cuenta a Estados Unidos han demostrado que ese retorno puede convertirse en una experiencia traumática para los grupos domésticos, que pueden quedar separados por tiempo indefinido.

Frente a ese escenario, se ha generalizado un arreglo entre hermanos que consiste en que los que están en Estados Unidos envían dinero para pagar a una cuidadora y alguna de las hermanas se encarga de organizar la logística de la atención a los padres en el lugar de origen. Por el cuidado de un anciano durante ocho o nueve horas durante el día se cobran 1500 pesos semanales. Una cantidad similar se paga en caso de que sea de noche. Es un arreglo costoso, pero funcional, en tanto los padres viven.

\section{La costura}

La reaparición de la maquila. Como ya se mencionó, el abaratamiento de la mano de obra en México ha vuelto a hacer rentable la maquila. Por ese motivo han surgido, en diferentes municipios y localidades de los Altos, talleres maquiladores para la confección de prendas de vestir, calzado y artículos para el hogar. Los materiales y las telas provienen de China y Corea y se confeccionan en la región. Así, se ha renovado la articulación entre talleres y trabajo a domicilio en la confección de una variedad de prendas de vestir, sábanas, cortinas, colchas, cobijas, manteles, edredones, toallas, juegos de baño, calzado.

Los talleres maquiladores trabajan para establecimientos industriales y comerciales, grandes y medianos, de ciudades como Aguascalientes, Ciudad de México, Guadalajara, Tepatitlán, Villa Hidalgo, Zapotlanejo. Desde allí se envía la confección de prendas y calzado a talleres maquiladores que se ubican en ranchos, pueblos y ciudades. Algunos, a su vez, distribuyen máquinas y envían trabajo a domicilio.

A cada taller se le destinan lotes de piezas cortadas de algún producto para coser, que es la etapa que requiere de más conocimiento, tiempo y trabajadores. Las dueñas de 
los talleres pueden acudir a recoger los lotes, lo que les da la posibilidad de escoger lo que quieren hacer, o bien se los llevan a los talleres y después los recogen.

Se trata de lotes pequeños, de artículos y modelos que cambian cada semana. Los talleres conocen y se adaptan a las fluctuaciones del trabajo maquilador: el año comienza mal, pero por lo regular las empresas todavía les deben dinero; se recuperan en febrero y les va bien hasta mayo, temporada alta que se cierra antes del 10 de mayo (Día de las Madres); entre mayo y agosto los pedidos se estancan; se recuperan si fabrican algún producto escolar; finalmente, de septiembre a diciembre es la mejor temporada, el invierno y las fiestas navideñas a las que llegan, gracias a Dios, como dicen, con mucho trabajo.

Encontramos actividades a domicilio, ligadas a industrias y establecimientos comerciales, en especial, en ranchos alejados. En Jalostotitlán se producen guantes industriales y se fabrican pantalones; en ranchos de Tepatitlán y Jesús María se cosen zapatos de hombre y de mujer. Se trata de empresas, con base regional, que entregan al trabajo a domicilio la fase más ardua de la confección del calzado: la costura a mano de la suela. Hay que decir que el calzado cosido tiene un costo más elevado que el pegado, por lo cual resulta rentable enviarlo a domicilio.

Las empresas tienen cuatro o cinco centros de distribución de trabajo a domicilio. En cada caso, una señora del rancho es la encargada de recibir los lotes de pares de zapatos y de distribuirlos a su red, de alrededor de diez mujeres, que acuden a recogerlos para coserlos en sus domicilios. Hoy en día, dicen las trabajadoras, la producción es muy variable y el pago depende del modelo de que se trate. El pago por la costura a mano de un par de zapatos puede variar entre 7 y 13 pesos y una trabajadora puede realizar entre 5 y 11 pares diarios si trabaja todo el día. Las mujeres trabajan sentadas en pequeños bancos y se cubren los dedos con dedales de piel, confeccionados por ellas, debido a la presión que deben hacer con la aguja. El trabajo a domicilio, reconocen las trabajadoras, está mal pagado pero lo aceptan en la medida en que no hay otros empleos ni trabajos en sus localidades. Cualquier otra opción laboral resulta más atractiva para las mujeres, en especial, para las jóvenes y las que tienen más educación. Como quiera, ese ingreso amortigua la inestabilidad de los ingresos de los esposos, muchos de ellos jornaleros.

La costura en la actualidad. Frente a los cambios acelerados que han experimentado la producción, la comercialización y, sobre todo, el consumo de la sociedad mexicana, la costura ha tenido que redefinirse, una vez más. Y lo ha hecho en tres sentidos: se ha susci- 
tado una nueva — y distinta — fragmentación de la producción; ha habido un viraje hacia la producción de prendas y artículos de moda; finalmente, se han desarrollado nuevas técnicas y productos. Lo común es que siguen siendo actividades que llevan a cabo tanto en los hogares como en talleres.

Las técnicas de bordado y tejido que se practican en la región son muy variadas pero en todas se reconoce un origen europeo: punto de cruz, deshilado, filigrana, rococó, trapeo, nudo francés, punto fino. En los últimos años han cobrado fuerza los trabajos con técnicas novedosas como listón y listón tejido, bombón, bordado en máquina.

Sin pretender ser exhaustivas, puede decirse que el punto de cruz predomina en los municipios de Tepatitlán y Acatic; el gancho en la microrregión de Arandas y el deshilado en los municipios del norte de la región, más cercanos a San Juan de los Lagos y Aguascalientes, los dos centros tradicionales de captación de ese tipo de producción. Hay especializaciones microrregionales. El frivolité se reconoce como la especialidad de San Ignacio Cerro Gordo. Otro lugar reconocido por la calidad y variedad de sus técnicas es Capilla de Guadalupe: trapeo, filigrana, deshilado, «voy y vengo», flor de guayabo, empavonado.

Las principales técnicas de tejido son: crochet (lo que llaman gancho), frivolité, dos agujas. El crochet y el gancho se utilizan para hacer cobijas, carpetas, zapatos, gorros, vestidos, blusas y bufandas. El crochet y el gancho se usan también para cerrar las prendas, es decir, darles el acabado de las orillas a servilletas, manteles, carpetas. El frivolité, una técnica laboriosa y costosa, se utiliza para hacer carpetas de diferentes tamaños, blusas, rebozos, adornos para el pelo y, cada vez más, accesorios: aretes, collares y pulseras. Las dos agujas sirven para confeccionar suéteres, gorros, bufandas, bolsas, pero sobre todo, cobijas grandes y de bebé.

Un primer cambio importante se ha dado en la elaboración de artículos tradicionales como las servilletas, manteles, carpetas, caminos (tela deshilada o bordada que se coloca al centro de la mesa sin cubrirla totalmente). Antes, como ya se mencionó, las trabajadoras elaboraban toda la prenda, hasta terminarla y entregarla para la venta. Lo que se observa ahora es la fragmentación del trabajo en un proceso donde diferentes mujeres, en sus casas, llevan a cabo las diversas tareas que conforman una prenda. Así, una mujer en un rancho puede hacer el deshilado, filigrana, trapeado o estampado que sirven de marco del artículo por confeccionar; luego pasa a otra mujer, que puede ser de otro lugar, quien le añade el punto de cruz; de ahí, puede irse a una tercera fase, donde otra señora le añade algún trabajo de filigrana; y puede ser transferido a otra mujer que hará el tejido a crochet 24 | 
o gancho de la orilla del mantel. Las comerciantes que encargan manteles suelen poner la tela, pero las trabajadoras prefieren comprar los hilos.

En ese caso, se dice que el artículo en cuestión tiene cuatro «trabajos», y eso determina su precio. La prenda terminada todavía debe ser sometida a las etapas de lavado, almidonado y planchado, que son las que le dan vista, es decir, las que estimulan la venta. Estas etapas pueden ser enviadas a trabajadoras a domicilio o la comerciante contrata a alguien para que, en su casa, las lave y planche. $\mathrm{O}$ bien, y esto es frecuente, la comerciante se encarga de las fases de lavado, almidonado y planchado. Esto significa que una prenda puede pasar por el trabajo a domicilio de entre dos y siete mujeres.

Hay mujeres que solo realizan el «cuadro», es decir, el bordado del centro de las piezas. Esto es frecuente en la confección de servilletas, tortilleros, carpetas, colchas para bebé. Esos cuadros deben ser sometidos a las etapas de armado, acabado, lavado y planchado. Muchas trabajadoras tienen acuerdos permanentes con alguna comerciante que termina las prendas y las vende o las entrega a otras comerciantes en tianguis y tiendas. También hay señoras que producen cuadros de manera independiente y cuando acumulan una cantidad conveniente para ellas, los llevan a vender al tianguis, las tiendas o con alguna conocida que se dedica a terminarlos y venderlos.

La confección de cuadros es más barata en los ranchos. Pero depende de la calidad y cantidad de trabajo que contengan. Las comerciantes reconocen y aprecian el tamaño de las puntadas, del punto de cruz, el reverso de las piezas, detalles que le añaden o quitan valor a una prenda. En 2014 se pagaba entre 16 y 23 pesos por el trabajo de bordar a mano un cuadro de servilleta. Un cuadro de colcha para bebé se pagaba entre 50 y 65 pesos, quehacer que requiere dos días de trabajo.

Otro trabajo fragmentado es el bordado a máquina. En él las mujeres elaboran el bordado de almohadones y colchas. Ellas hacen el cuadro, es decir, la parte bordada que, posteriormente, se integra a la pieza mayor y se envía a otra mujer para ser acabada — cosida, tejida - y así pasar a las fases de lavado, almidonado y planchado. Existe bastante libertad para escoger los diseños y colores de los bordados, aunque también se aceptan pedidos especiales.

Hay bordadoras que reciben la tela, otras prefieren comprarla; pero, en general, todas compran los hilos porque así tienen muchos colores, maximizan ese material y calculan mejor sus costos. El bordado a máquina es, obviamente, más rápido que a mano. Una trabajadora experimentada puede hacer doce pares de almohadones al día. En marzo de 2014 se pagaba a 16 pesos el cuadro de un par de almohadones bordados, lo cual quiere 
decir que una bordadora percibe 234 pesos diarios. Si trabaja a ese ritmo, que suele ser lo normal, una bordadora a máquina recibe en cinco días de trabajo 1170 pesos, un ingreso similar al de una trabajadora en una granja, que gana 1200 pesos. En este caso, la trabajadora asume los costos de la máquina, sus arreglos y mantenimiento.

Un segundo cambio es la innovación permanente motivada por la moda. Se trata de elaborar artículos, prendas, accesorios atractivos y que se estén usando de tal manera que tengan mayor demanda que los tradicionales. Esto ha sido posible sobre todo en el bordado a máquina. Las trabajadoras, por iniciativa propia, pero también por la demanda de las compradoras, han introducido diseños novedosos en los cuadros de las almohadas, cojines, colchas para bebés y niños: imágenes de animales, personajes de caricaturas, series y películas, paisajes con escenas simpáticas y bucólicas. Ese cambio ha tenido un impacto positivo en la demanda y el ingreso de las bordadoras a máquina.

Las tejedoras han procurado incorporar la moda y dirigirse a los públicos juvenil e infantil tanto en el tipo de prendas como en el diseño y los colores. Así, han comenzado a tejerse blusas, chalecos, vestidos, rebozos, chales, bufandas, cinturones, bolsas, accesorios como aretes, ballerinas para el pelo, brazaletes, broches y pasadores, collares tejidos a crochet, gancho, frivolité y dos agujas.

Se ha incrementado la fabricación de todo tipo de «morrales», como los llaman, de todas las técnicas y para todos los usos: paneras, portakleenex, portabotellas de agua, portacelulares. Por lo regular, las tejedoras compran los materiales, deciden cómo hacer el producto, los colores y la decoración. Las demandas cambiantes del mercado les son transmitidas por las comerciantes, por amigas y vecinas que les hacen encargos y ellas mismas experimentan con productos y estilos.

Un producto de gran demanda y que ha detonado el trabajo a domicilio son los juegos de baño de tres piezas, que se pueden encontrar bordados a mano, a máquina, tejidos con diferentes técnicas, en combinaciones de bordado y tejido. Hay jóvenes que producen juegos de baño que, a través de una densa red de producción a domicilio, abastecen negocios y tiendas de departamentos de la Ciudad de México.

En tercer lugar, las jóvenes han inventado o combinado técnicas y productos. Gracias a habilidades y saberes aprendidos en los hogares o en las clases de manualidades del D I F, ellas han inventado técnicas como el pintado de prendas o la confección de artículos de pasta. Han hecho mezclas de técnicas para ofrecer artículos novedosos y atractivos: bolsas de rafia, tortilleros, sábanas, almohadas de tela adornadas con listón, marcos para fotografías, adornos para el pelo, aretes y collares, diferentes figuras y escenas de pasta. 
Se confeccionan bajo pedido de comerciantes que los venden en tianguis y tiendas, pero también reciben encargos para regalos y eventos especiales. Las figuras de pasta o los trabajos de listón son muy cotizados para regalar como bolos de fiestas de cumpleaños, boda, XV años. Una joven puede recibir, por ejemplo, el encargo de confeccionar 50 marcos de listón para regalar en una fiesta de cumpleaños.

Los productos de la costura se venden, al mayoreo y menudeo, en tres lugares. En primer lugar, en el tianguis de Tepatitlán, que se celebra los días jueves y domingo; en las tiendas establecidas y los puestos semifijos de San Juan de los Lagos, y ahora también en Santa Ana de Guadalupe, donde se venera a Santo Toribio Romo, en el municipio de Jalostotitlán. En los tres lugares hay cada vez más productos industriales de origen chino, pero abundan sobre todo en San Juan de los Lagos y en Santa Ana de Guadalupe.

Son mercados de productos de bajo precio que tienden a decrecer, más que a crecer. Una clientela importante han sido las migrantes en Estados Unidos. Los artículos de costura, baratos, que podían ser transportados en grandes cantidades, se convirtieron en uno de los principales productos de exportación de la región. Los migrantes acudían al tianguis, las tiendas, o hacían encargos a comerciantes y trabajadoras, de servilletas, manteles, carpetas, caminos que se llevaban en las camionetas para ofrecer como regalo o para vender en sus lugares de destino en Estados Unidos. Los bordados a mano forman parte del mercado de la nostalgia, es decir, de los productos añorados por los migrantes, pero que también han sido revalorizados por ser artesanías hechas a mano, atributo altamente valorado y bien retribuido en Estados Unidos.

Los principales compradores de la costura tradicional y de los nuevos artículos y objetos son comerciantes de tianguis y mercados de ciudades como Aguascalientes, Colima, Guadalajara, León, San Juan de los Lagos, Villa Hidalgo, y de lugares indefinidos de los estados de Guanajuato, Jalisco, Nayarit y Puebla.

En el tianguis de Tepatitlán se advierte un ejemplo de los circuitos y valores de los objetos hechos a mano: cada semana, indígenas purépechas de Michoacán recorren los puestos y compran por mayoreo servilletas bordadas, de no muy buena calidad pero de muy bajo precio, para venderlas como artesanías purépechas en espacios turísticos de Michoacán y Jalisco. Así, los productos tradicionales forman parte no solo del mercado de la nostalgia sino también del mercado étnico-turístico, de gran dinamismo en la actualidad.

Los productos de la costura de los Altos, aunque de muy alta y variada calidad técnica, son de diseños tradicionales y de gusto popular, de bajo costo y bajo precio; por debajo de los textiles de origen indígena. La producción alteña carece de un plus étnico, 
que tanto se valora actualmente. Tampoco han llegado a la región, como ha sucedido con la producción indígena, diseñadores interesados en ese tipo de producción manual.

\section{El trabajo: una necesidad femenina permanente}

Lo anterior sugiere que la intensa y permanente participación de las mujeres en los empleos y trabajos que les procuren ingresos de manera regular es una característica central de la condición femenina en los Altos de Jalisco. Hoy por hoy, las mujeres de todas las edades y condiciones conyugales y familiares desempeñan uno o varios quehaceres, dentro o fuera del hogar, por los que reciben salarios o ingresos.

Aunque, como se ha mostrado, en la región existe una vigorosa y antigua tradición de trabajo femenino, la búsqueda actual de ingresos por parte de las mujeres está relacionada con cuatro circunstancias que detonaron en la década de 1990.

En primer lugar, la pérdida de la condición de proveedor de los hombres. En la actualidad, los hombres, en especial los jóvenes, carecen de tierras y, en muchas ocasiones, ya no cuentan con un solar o casa propia. Aunque hay arrendatarios de parcelas y medieros de ranchos, lo que más abunda es el trabajo jornalero. Son trabajadores que viven de lo que perciben cada ocho días pero hay semanas en las que no los contratan. El trabajo masculino se ha vuelto precario, inestable y mal pagado. Se ha precarizado hasta poner en duda su papel como proveedores únicos, principales o permanentes de sus hogares.

De hecho, la categoría laboral que más se ha extendido en el mundo rural y los espacios periurbanos es el jornalerismo como inserción laboral masculina a largo plazo. Con datos de la Encuesta Nacional de Empleo se ha constatado que las remuneraciones por hora y por semana de los sujetos agropecuarios resultaban menores y la proporción de asegurados era muy inferior a la de los que se dedicaban a actividades no agropecuarias (Pacheco, 2010). El jornalerismo, como forma de inserción laboral masculina, es una forma de empleo eventual que se lleva a cabo en las labores agropecuarias, pero también en la construcción, los servicios de carga y descarga, el transporte.

En segundo lugar, y muy ligado a lo anterior, los miembros de los hogares comparten la situación y la certeza de que no pueden vivir de un solo ingreso, menos aún si este proviene del jornalerismo. Aunque con resistencias discursivas, ha quedado atrás el prohibirles a las mujeres, ya sean esposas, hijas o hermanas, que trabajen. El derecho al trabajo, una reivindicación de las obreras de la década de 1970 (Rosado, 1990) ha dejado de serlo y se ha convertido en una obligación de las mujeres. 
Las jóvenes han perdido el derecho a permanecer en los hogares sin trabajar ni procurarse ingresos en tanto se unían y salían de la casa de sus padres, como sucedía hasta hace no mucho tiempo. En la situación actual, si dejan de estudiar y pierden la beca de Prospera, por ejemplo, deben emplearse fuera del hogar o conseguir algún ingreso: trabajo a domicilio, comercio, servicio; lo que sea que incorpore o redistribuya dinero en el interior de los hogares.

De esta manera, las solteras, madres solteras o las que han regresado a los hogares de sus padres porque se han separado o han sido «dejadas», han pasado a ser activos imprescindibles para asegurar los ingresos de una casa frente a la irregularidad de los trabajos y salarios masculinos y la precariedad de los grupos domésticos en general.

Eso quiere decir, en tercer lugar, que el ingreso de las mujeres, por las vías que sean, se ha vuelto indispensable para la sobrevivencia de los hogares. Y, aunque reducido y fluctuante, puede ser más estable y permanente que el de los hombres. Existe hoy una aceptación social y familiar generalizada de que son imprescindibles los ingresos femeninos en los hogares. Sin embargo, las cargas de trabajo doméstico y el cuidado de los hijos siguen siendo obligación primordial de las mujeres.

Hay que decir que todavía hay mujeres que alientan la idea del proveedor masculino e insisten en minimizar su trabajo. Prefieren insistir en que ellas trabajan «por pasar el tiempo»y que su aportación económica es «muy poquita». En ellas persiste el discurso tradicional de la cultura patriarcal de que «al hombre hay que darle su lugar», aunque se contradiga con su experiencia cotidiana. Pero otras ya reconocen el papel central de su aportación económica al hogar, en especial cuando se trata de maridos flojos o desobligados. Las jóvenes, en especial, entienden que todas las actividades que realizan, dentro y fuera de los hogares, son trabajo y que, por lo tanto, requieren de retribución económica y derechos en el interior de los hogares.

En cuarto lugar, la obligación femenina de trabajar parecería estar relacionada con la reducción de las remesas. Un ingreso centenario de los hogares de la región fueron las remesas que enviaban los hombres cuando eran migrantes en Estados Unidos. Maridos e hijos enviaban dinero de manera regular a sus hogares con el fin de construir o arreglar la casa, comprar tierras, animales, instalar un negocio (Massey et al., 1991).

Sin embargo, las transformaciones en la condición de los migrantes en Estados Unidos y el nuevo patrón migratorio han trastocado ese escenario. Hoy por hoy, el flujo migratorio, documentado e indocumentado, a Estados Unidos está prácticamente cancelado (Durand, 2013). Los cambios sociodemográficos y el control fronterizo han mo- 
dificado el carácter circular tradicional de la migración mexicana de tal manera que la posibilidad de los jóvenes de ir a trabajar a Estados Unidos es prácticamente nula (Durand y Arias, 2014). En 1988 en más de la mitad de los hogares de la región había miembros con experiencia migratoria en Estados Unidos, proporción que se redujo a menos de una tercera parte (30.80\%) en 2014-2015 (Arias, 2016). Aunque la proporción sigue siendo elevada en comparación con la media nacional (alrededor de $10 \%$ ), la tendencia es indudablemente a la baja.

Esto ha tenido tres consecuencias que han afectado la recepción de remesas en los hogares. En primer lugar, los migrantes que desde 1986, con la amnistía promovida por la I RC A, legalizaron su residencia en Estados Unidos (Durand y Massey, 2003) contrajeron compromisos económicos — compra de casas, establecimiento de negocios-y obligaciones familiares - matrimonio, hijos, nietos - en el otro lado. Los intereses y las obligaciones con sus hogares de origen se han reducido de manera paulatina, quizá poco deseada por los migrantes en Estados Unidos y sus familias en México, pero imparable. Los migrantes han especializado y espacializado el envío de remesas a los hogares de sus padres en México. Ahora envían dinero para celebraciones especiales, como el Día de las Madres, Navidad o cumpleaños, pero sobre todo cuando los padres se enferman y requieren de medicamentos, atención médica, equipo y servicios especializados. Pero han dejado de enviar dinero cada quince días o cada mes como sucedía antes.

En segundo lugar, ante la imposibilidad de emigrar a Estados Unidos, se ha intensificado la salida a destinos nacionales, en especial de los jóvenes, casados y solteros, que eran los que enviaban más remesas a los hogares de sus padres. Al disminuir el número de migrantes se ha reducido la posibilidad de que los hogares tengan ingresos regulares por esa vía. Y los jóvenes que han emigrado a ciudades de la región y fuera de ella no ganan lo suficiente como para enviar dinero a sus padres, como sucedía cuando emigraban a Estados Unidos.

En tercer lugar, se ha intensificado el proceso de disolución de uniones. La permanencia prolongada e indefinida de los hombres en Estados Unidos, otro efecto del modelo migratorio actual, ha llevado a la ruptura de uniones establecidas en los lugares de origen. Muchos migrantes han dejado de responsabilizarse y enviar dinero a las esposas y a los hijos que procrearon en México.

En estas condiciones, con ingresos masculinos mermados y la reducción o el cese de remesas, la contribución de los salarios e ingresos femeninos ha cobrado una enorme centralidad en la integración de los presupuestos de los hogares. Además de la necesidad 
económica, se ha observado que la permanencia, intensificación, recreación y reinvención de empleos y trabajos por parte de las mujeres están asociadas a cuatro situaciones que han dado lugar a cambios en la condición femenina.

En primer lugar, la dificultad o el desencanto por las condiciones de trabajo e ingresos de los establecimientos o instituciones que ofrecen prestaciones sociales ha contribuido al surgimiento, la permanencia o la invención de trabajos por cuenta propia, de manera individual o mediante la creación de pequeñas empresas. Aunque se trata de una proporción menor, hay jóvenes, varias de ellas universitarias, que han optado por permanecer, pero sobre todo por incursionar en negocios familiares.

En la región existen experiencias de migración interna y actividad empresarial que eran muy masculinas y han sido retomadas por las jóvenes. Desde hace décadas los vecinos de la pequeña localidad de Santiaguito, del municipio de Arandas, han emigrado para dedicarse a las taquerías, y los de San Ignacio Cerro Gordo para abrir tiendas de abarrotes en las ciudades, en especial, en la capital del país (Arias, 2017; Muñoz y Sánchez, 2017). En la actualidad, las mujeres, en especial las jóvenes, se han incorporado como propietarias, copropietarias y trabajadoras de taquerías y tiendas ya no solo en las ciudades de México y Guadalajara sino en muchas ciudades, medias y pequeñas, de todo el país.

En segundo lugar, a la decisión femenina de no formar hogares a edad temprana. Como ya se ha señalado, las jóvenes han perdido el derecho a no generar ingresos mientras permanecen en sus hogares de origen y en la región es muy difícil que una mujer soltera viva sola. El empleo en empresas, instituciones o la dedicación a actividades por cuenta propia les han permitido permanecer solteras más allá de las presiones familiares y de propuestas matrimoniales no deseadas.

En tercer lugar, el trabajo y los ingresos femeninos han apoyado la disolución de uniones por parte de las mujeres, en especial cuando tienen hijos pequeños. El trabajo les ha permitido separarse de maridos flojos, desobligados o violentos. Las mujeres, con ingresos regulares, aunque sean precarios, pueden hacerse cargo de sus hijos, aunque esto suponga tener que regresar a sus hogares de origen, de manera temporal al menos.

En cuarto lugar, el trabajo ha facilitado la disolución de uniones de mujeres en fases tardías de sus vidas. La mujer trabajadora, con hijos mayores que ya no dependen de ella, pero que la apoyan, puede tomar la decisión de salir de una unión desafortunada.

Desde luego que no solo el trabajo, donde quiera y como quiera que sea, contribuye a hacer posibles estos cambios. Pero la posibilidad de contar con ingresos propios, aunada a nuevos escenarios, prácticas y discursos sobre la condición femenina, les han permi- 
tido tomar decisiones personales que hasta hace poco tiempo resultaban imposibles en la región.

\section{En síntesis}

Los empleos y trabajos de las mujeres que se han mantenido, surgen, se recrean y reinventan en regiones particulares, como los Altos de Jalisco, hay que entenderlos de manera dinámica y en relación con las circunstancias económicas y los escenarios sociales y familiares actuales, pero siempre cambiantes.

Los empleos y trabajos descritos son expresión de las transformaciones recientes en el comportamiento de los sectores económicos y los mercados de trabajo que forman parte de la globalización, pero también de las maneras como las mujeres los han captado $y$ transformado en nichos laborales que les permiten sobrevivir en términos económicos y manejar las transiciones y relaciones de sus hogares.

La incorporación de las mujeres al trabajo y a la búsqueda de ingresos ha detonado cambios en las condiciones, horarios, hábitos, divisiones del trabajo en los hogares que desafían los arreglos jerárquicos tradicionales basados en la jefatura y la proveeduría masculinas. El cuidado de niños y ancianos y las necesidades de alimentación y arreglo personal de las trabajadoras se han convertido en ventanas de oportunidad para el surgimiento de servicios a cargo de mujeres. Ellas siempre están buscando lo que se puede hacer para convertir en ingresos los cambios económicos y sociales que viven sus entornos sociales y familiares. No siempre resulta bien ni a la primera, pero la cantidad de ejemplos viables constata que ha sido y sigue siendo posible descubrir nichos laborales o de negocios.

Los trabajos de las mujeres, dentro y fuera de la casa, han ayudado a solventar cambios irreversibles de los hogares: la precariedad e irregularidad de los empleos e ingresos masculinos; la imposibilidad de vivir de un solo ingreso y que este sea el ingreso masculino; la presión para que ellas generen ingresos sin descuidar sus obligaciones tradicionales. El trabajo, donde quiera que sea, les ha permitido a las mujeres generar ingresos sin desatender el hogar ni a los hijos pequeños. Eso parece muy tradicional.

Pero les ha permitido hacer frente y manejar otras transiciones inesperadas también irreversibles. En especial una: la disolución de las uniones, tanto porque han sido «abandonadas» como también porque ellas mismas han decidido salir de relaciones desafortunadas. Y eso no es poca cosa $\diamond$ 


\section{Referencias}

Anda, F. de (1989). Pinceladas de sabor local. México: Editor Gustavo de Anda.

Arias, P. (2017). Entre dos crisis. Los abarroteros de San Ignacio Cerro Gordo. En Arias, P. (coord.), Migrantes exitosos. La franquicia social como modelo de negocios (pp. 150-170). Guadalajara: Universidad de Guadalajara-Centro Universitario de Ciencias Sociales y Humanidades.

Arias, P. (2016). Del permiso a la obligación. El trabajo femenino en los Altos de Jalisco. Papeles de Población, 22 (90), 197-228.

Arias, P., Sánchez García, I. y Muñoz Durán, M. (2015). Quehaceres y obras. El trabajo femenino en los Altos de Jalisco. Guadalajara: Secretaría de Cultura del Gobierno del Estado de Jalisco.

Arias, P. (2009). Del arraigo a la diáspora. Dilemas de la familia rural. México: Miguel Ángel Porrúa, Centro Universitario de Ciencias Sociales y Humanidades.

Arias, P. y Wilson, F. (1997). La aguja y el surco. Cambio regional, consumo y relaciones de género en la industria de la ropa en México. México: Universidad de Guadalajara, Centre for Development Research.

Arias, P. (1988). La pequeña empresa en el occidente rural. Estudios Sociológicos, vi (17), 405436.

Bataillon, C. y Rivière D’Arc, H. (1973). La ciudad de México. México: Sepsetentas.

Díaz, J. y Rodríguez, R. (1979). El movimiento cristero. Sociedad y conflicto en los Altos de Jalisco. México: C I S - I N A H, Nueva Imagen.

Durand, J. (2013). Nueva fase migratoria. Papeles de Población, 19(77), 83-113.

Durand, J. y Arias, P. (2004). La vida en el Norte. Historia e iconografía de la migración MéxicoEstados Unidos. Guadalajara: Universidad de Guadalajara.

Durand, J. y Massey, D. S. (2003). Clandestinos. Migración México-Estados Unidos en los albores del siglo XXI. México: Universidad Autónoma de Zacatecas, Miguel Ángel Porrúa.

Gandini, L., Padrón Innamorato, M. y Navarrete, E. L. (2017). Presentación. En Padrón Innamorato, M., Gandini, L. y Navarrete, E. L. (coords.), No todo el trabajo es empleo. Avances y desafíos en la conceptualización y medición del trabajo en México (pp.15-22). México: El Colegio Mexiquense, Universidad Nacional Autónoma de México-Instituto de Investigaciones Jurídicas.

García, B. y Oliveira, O. de (2011). Family changes and public policies in Latin America. Annual Review of Sociology, 37, 593-611.

García Guzmán, B. (2010). Población económicamente activa: evolución y perspectivas. En García, B. y Ordorica, M. (coords.), Los grandes problemas nacionales. I. Población (pp. 363-392). México: El Colegio de México. 
Garza, G. (1980). Industrialización de las principales ciudades de México. México: El Colegio de México.

González, L. (1989). Gente del campo. Vuelta, 151, 22-29.

González Montes, S. y Salles, V. (1995). Mujeres que se quedan, mujeres que se van ... Continuidad y cambios de las relaciones sociales en contextos de aceleradas mudanzas rurales. En González Montes, S. y Salles, V. (coords.), Relaciones de género y transformaciones agrarias (pp. 15-50). México: El Colegio de México.

Jiménez Restrepo, D. M. (2012). La informalidad laboral en América Latina: ¿explicación estructuralista o institucionalista? Cuadernos de Economía, X X XI (58), 113-143.

Massey, D. S. et al. (1991). Los ausentes. El proceso social de la migración internacional en el Occidente de México. México: Conaculta.

Muñoz Durán, M. y Sánchez García, I. (2017). La evidencia del éxito. Residencias y mausoleos en Santiaguito, Arandas, Jalisco. En Arias, P. (coord.), Migrantes exitosos. La franquicia social como modelo de negocios (pp. 100-147). Guadalajara: Universidad de Guadalajara-Centro Universitario de Ciencias Sociales y Humanidades.

Pacheco Gómez, E. (2010). Evolución de la población que labora en actividades agropecuarias en términos sociodemográficos. En García, B. y Ordorica, M. (coords.), Los grandes problemas nacionales. I. Población (pp. 393-429). México: El Colegio de México.

Padrón Innamorato, M., Gandini, L. y Navarrete, E. L. (coords.) (2017). No todo el trabajo es empleo. Avances y desafíos en la conceptualización y medición del trabajo en México. México: El Colegio Mexiquense, Universidad Nacional Autónoma de México-Instituto de Investigaciones Jurídicas.

Rollwagen, J. (1968). The paleteros of Mexticacán, Jalisco. A study of entrepreneurship in Mexico. Ph. Dissertation, University of Oregon-Department of Anthropology. Oregon.

Roberts, B. (1978). Cities of peasants. California: Sage Publications.

Rosado, G. (1990). De campesinas inmigrantes a obreras de la fresa en el valle de Zamora, Michoacán. En Mumert, G. (ed.), Población y trabajo en contextos regionales (pp. 45-71).Zamora: El Colegio de Michoacán.

Taylor, P. S. (2013). Arandas, Jalisco: una comunidad campesina, 1931-1932. En Arias, P. y Durand, J. (invest. y ed.), Paul S. Taylor y la migración jalisciense a Estados Unidos (pp. 67-185). México: Universidad de Guadalajara-Centro Universitario de los Altos.

Tokman, V. (s.f.). De la informalidad a la modernidad. Boletín Cinterfor, 155.

Wilson, F. (1990). De la casa al taller. Zamora: El Colegio de Michoacán.

Yáñez, A. (1947 [1996]). Al filo del agua. México, Editorial Porrúa. 